\title{
Unified Framework to Select an loT Platform for Industrial Energy Management Systems
}

This paper was downloaded from TechRxiv (https://www.techrxiv.org).

\section{LICENSE}

CC BY 4.0

SUBMISSION DATE / POSTED DATE

$12-11-2021 / 18-11-2021$

\section{CITATION}

Ullah, Mehar; Narayanan, Arun; Wolff, Annika; Nardelli, Pedro (2021): Unified Framework to Select an loT Platform for Industrial Energy Management Systems. TechRxiv. Preprint.

https://www.techrxiv.org/articles/preprint/Unified_Framework_to_Select_an_loT_Platform_for_Industrial_Energy

$\mathrm{DOI}$

10.36227/techrxiv.16955323.v1 


\title{
Unified Framework to Select an IoT Platform for Industrial Energy Management Systems
}

\author{
Mehar Ullah, Arun Narayanan, Annika Wolff, Pedro H. J. Nardelli \\ LUT University, Lappeenranta, Finland \\ mehar.ullah@lut.fi
}

\begin{abstract}
The world population is increasing at a rapid speed and so are the requirements for everyday life like energy, technology, and industries. In the past few years, there has been a huge increase and development in the industrial sector and new technology like the Internet of Things (IoT) has played a major role in it. The industrial sector is consuming a large portion of the energy worldwide and is contributing a large amount of greenhouse gas emissions. Hence, they face significant economic, social and environmental pressures to create energy-efficient processes and systems of production and directly manage their energy consumption, looking at aspects beyond direct costs. One of the effective ways to reduce energy consumption, cost, and greenhouse gas emissions is to use an industrial energy management system (IEnMS). The IEnMS uses IoT for (big) data collection, processing, storing, and visualization. In this case, one key challenge the industries face is the selection of an IoT platform among the hundreds of IoT platforms in the market. In this paper, we adapt our previously proposed general framework to choose an IoT platform focusing here on the requirements of IEnMS. The proposed framework provides an objective methodology that can be used to select the most suitable IoT platform for different IEnMS based on their particular requirements.
\end{abstract}

Keywords-IoT, IoT platform, platform features, industrial energy management system, framework

\section{INTRODUCTION}

Energy and climate change are one of the most important topics in the public eye. Across every sector whether it is industry, housing or government, there is an attempt to save energy and control climate changes [1] because of the fact that energy is finite and can be used in a smart and efficient way to protect the current and future generations [2]. Industry is the one that is consuming the highest amount of energy and it is estimated that about $42.3 \%$ of the total energy produced in the world is consumed by the industrial sector [3]. For a company to be in the race of competition and position themselves as a market leader in the long run, the company has to give priority to the management and operation of the company's energy system. Due to this reason industry needs more attention to use effective energy management.

Energy management is one of the promising means for an industry to reduce energy consumption and related energy cost and reduce carbon emission. Back in nineties, energy cost was a small factor for the industrial companies, this is due to the reason that the energy cost was very low as compared to the production size and greenhouse gas emission was not the priority. But within the last decades the energy and energy sourcing prices in Europe increased rapidly. Industrial sector is focusing on energy management in which the company prepares long term energy policies, planning and targeting for the energy use. Industrial Energy Management System (IEnMS) is used to implement this whole process and control energy consumption, green house emission, reduction in energy use by a specific percentage and save cost.

The Internet of Things (IoT) is promising to make the environment smarter and connecting devices to each other and with the platform for communication and data exchange. Connected devices are adopting IoT at a rapid pace. Since IoT is expanding continuously, the need for connectivity methods grows to ensure that the devices work, capture, analyze and manage the data accurately. For efficient connections and sharing of information, communications protocols like MQTT and HTTP are used [4] . The best way to store, process and analyse that data within its infrastructure is by using an IoT platform that comes with the data processing capabilities [5]. It will not only allow you to collect data from Industrial machines but can also help you set up a custom visualization dashboard.

This contribution aims at utilizing our developed theoretical framework for selecting a suitable IoT platform for IEnMS by using IoT and big-data. Our goal is to provide an objective while general methodology that different industries can apply when selecting the most suitable IoT platform for IEnMS based on their particular needs and business requirements. In other words, this paper will support industries to carry out a detailed analysis of their own energy requirements and understand the key components of their Energy Management (EnM) to find the best match for their IEnMS. The study will find the answers of the following questions. 1) What are the components of EnM in industrial sector? 2) How are the Industrial energy management systems working using IoT and big-data? 3) How to design a framework for selecting a suitable IoT platform for industrial energy management system by comparing the requirements of IEnMS with the features provided by the IoT platforms?

The rest of the paper is organized as follows. Sec. II contains Industrial EnM (IEnM). Sec. III explains IEnMS and in same section we have explained Industrial Energy Management system its working. Sec. IV contains Internet of Things (IoT), IoT platform and the need for IoT platform. Sec. V contains our proposed theoretical framework for selecting IoT framework for IEnMS. Sec. VI concludes the paper. 


\section{INDUSTRIAL ENERGY MANAGEMENT (IENM)}

One of the indicators of the economic development is the fast growth in industrial sector. With the passage of time the number of industries is growing and so is the need of energy [6]. The increase in the use of energy will be continuous with the rapid development in the industrial sector and the fast growth of the world population and will create problems like increase in energy price and the carbon emission. Industry needs energy management to use energy efficiently and also control carbon emission. Energy management consists of practices and processes to improve energy efficiency. As a result, in the recent years many organizations have put focus on energy related issues to improve their productions and operations and improve energy efficiency and thereby reduce energy usage and energy cost. Industry needs energy management to use energy efficiently and also control carbon emission.

\section{A. Components of IEnM}

In this section we will discuss the important components of IEnM that we have identified from the literature [6][10]. There are five main components in IEnM planning/strategy, operation/implementation, controlling, organization and culture.

Planning/Strategy: The first phase of EnM has three parts. The first part is the written long-term energy policy of the company. Second part is energy planning and target setting in which the industry is making the plans and setting future targets for the energy use. The target may be the lowering the green house gas emission, energy consumption, reduction in energy use by specific percentage etc.. Third part is the strategic energy risk management that is used to analyze any type of risk the company can face related to the energy use and how the risk can be managed by the company's predetermined financial objectives and risk tolerance.

Operation/Implementation: The second phase of EnM that consist of three parts. The first part is Implementation of energy efficiency measure in which the companies are implement specific energy projects and energy efficiency technologies to reduce electricity consumption. The second part is the investment decision on energy efficiency measure in which companies are conducting a systematic economic calculation to calculate the return of investment. The third part is the energy audit, in which the companies within the operations of an energy management constantly review the status-quo and highlight energy saving potential.

Controlling: The third phase of EnM that consist of three parts energy accounting, performance measurement and bench-marking. Energy accounting is the process of constant analysis of the energy consumption and measure the energy efficiency monitoring on a regular basis and is reported. Performance measurement is an integral part of EnM and it defines the key performance indicators (KPI) for energy efficiency. KPI's are describing the relationship between an activity and the required energy. Energy benchmarking is an activity that is focusing on energy perfor- mance and can be defined as the method used to compare the energy efficiency between or within entities and is a useful contribution to reduction in energy use and related cost and emission. There are three types of bench-marking industrial benchmark, historical benchmark and companywise benchmark. In industrial benchmark, the company compares its own facilities and process with the facilities and process of other company. In historical benchmarking the company compares its energy consumption of a process or facility with its own process or facility in the early times. In company-wise bench-marking the company compares its facilities and processes inside the company.

Organization: The fourth phase of energy management that consist of two parts. Selection of energy manager which is based on the experience and should be climate friendly. There should be a close link between the energy manager and the top management, and is responsible to update the top management about the activities and progress of the energy management. The second part is the integration and standardization. According to industrial companies energy management should be integrated with the production management processes using ICT tools and standardization. The production process and evaluation of potential energy saving investment can be controlled by the ICT tools and the transparency of the industrial companies can be increased by the standardization.

Culture: The last phase of energy management consists of two parts. First part is education and training. In industries at the corporate level or plant level the energy manager needs man-power which require basic education to meet the requirements. For this purpose, the industrial companies need continuous energy related training, which provides positive impacts on the energy management. Staff motivation is the second part in which the industrial companies needs to motivate the staff to actively participate in improving the energy efficiency. In that case the companies give rewards to the technical and operational staff, which helps to sustain the momentum and improve the overall support for the energy management program.

\section{FUNDAMENTALS OF IENMS}

For an organization to improve its energy performance, one of the best way is to use an Industrial Energy Management System (IEnMS). It monitors, controls, and optimizes energy performance in a plant and measures the consumption of energy. It is also used to diagnose problems like over-consumption and leaks across the entire plant. For energy consumers including industrial, public sector and commercial organizations an EnMS is a framework to manage their energy usage and can be defined as "a set of interrelated or interacting elements of a plan which sets an energy efficiency objective and a strategy to achieve that objective" [11]. it provides companies the opportunities to improve energy savings by adopting energy saving technologies. In most cases for a successful implementation of EnMS required low investment cost and specialized expertise and staff training. 
TABLE I

REQUIREMENTS OF INDUSTRIAL ENERGY MANAGEMENT [7]

\begin{tabular}{|c|c|}
\hline S No & Requirements for IEnM \\
\hline 1 & Development and implementation of strategic plan that includes the energy policy and specific targets for the energy savings. \\
\hline 2 & Organizing different energy activities including the allocation of responsibilities and tasks. \\
\hline 3 & Establishment of management team that is leading by an energy manager who will be responsible to report directly to the high management. \\
\hline 4 & Development of related policies and procedures which can include energy procurement, energy usage, cheap energy purchases etc. \\
\hline 5 & Carrying out the initial energy audit to identify energy saving potentials \\
\hline 6 & Planning and implementation of energy efficient measures \\
\hline 7 & Identification of the company's unique key performance indicators which can show the measure progress on a regular basis. \\
\hline 8 & Implementation of the energy meters for the monitoring of the energy consumption at the main production processes at a regular interval. \\
\hline 10 & Providing progress to the high management so that the management shows interest in the energy management activities. \\
\hline 11 & Training, motivating, and proving information to the employees of the company about energy management activities. \\
\hline
\end{tabular}

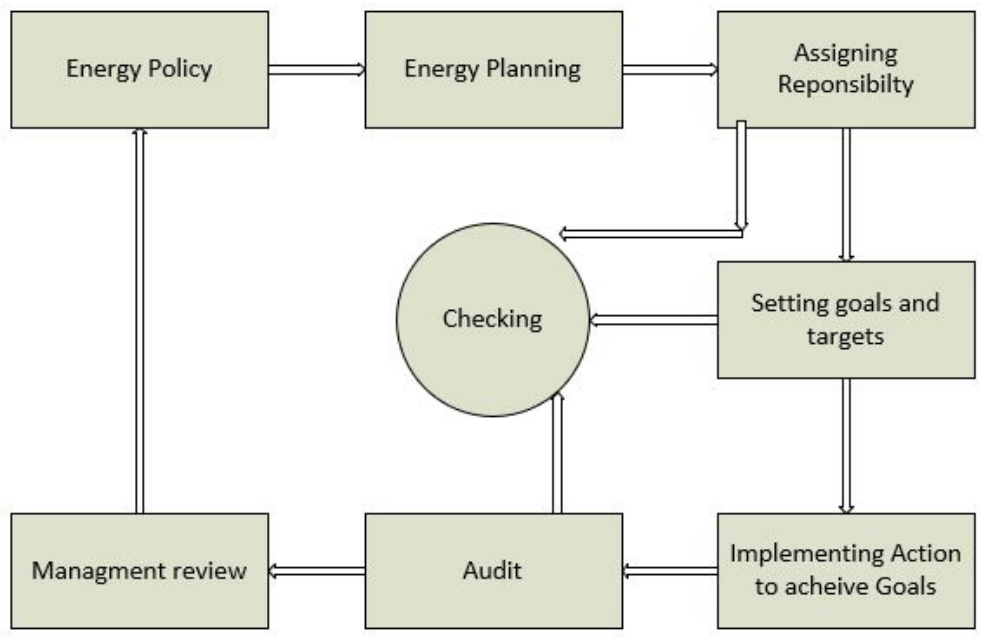

Fig. 1. Industrial Energy Management

Industries are required to follow a series of defined steps to establish a successful EnM. The main steps required are development of energy policy and assigning responsibilities, highlighting main energy users, setting measurable goals and targets, implementing actions to achieve the goals, checking actions are successful, and continuous system review as shown in Figure 1.

\section{A. Working of Industrial Energy Management System}

An Industrial Energy Management System is a computer-based system that is used to collect and measure energy data from the field like HVAC units (heating, ventilation and air-conditioning), lighting system, water and gas meters installed on production line etc. and provide that information to the user. The working of IEnMS is explained below.

Building data collection strategy: A system that is used to collect real time accurate and granular data and have the information that where and when the energy has been used and by which device(machine). The data is collected by installing sensors/smart meters, and sub-meters on the incoming supply and the large energy consumer (device). For example, on the HVAC, production line, boilers etc. The objective of this part is to monitor the collection of real-time data that shows where the majority of the energy being used.

Transform raw energy data into useful information: In this phase the collected data is analyzed, interpreted and is converted into useful information. Here the big-data software is used that can easily import the raw data from different machines using IoT devices and then convert the raw data into useful information in the forms of charts, graphs etc. that is user friendly. Here the raw data collected may related to the production levels, weather data, humanity and other factors that could influence energy usage to generate the key performance indicators.

Assign responsibility, analyze data: In this phase the information provided needs to be converted into useful and meaningful reports, that can only be possible by adding the information to the knowledge of the facility and some expertise in energy management. This can be done by the energy manager. The role of the energy manager is to interpret the information provided by the Industrial Energy Management System and combine this information with the company's process, set the targets.

Interpret the results, and agree an action plan: Here in this phase the energy usage reports are available to the energy manager. The energy manager and energy management team start communication with the departments to start an energy policy and make agree to an action plan.

\section{INTERNET OF THINGS AND IOT PLATFORM}

The term "Internet of Things", or "IoT", was introduced by the British technology entrepreneur Kevin Ashton in 1999 as the title of a presentation at Procter and Gamble. 


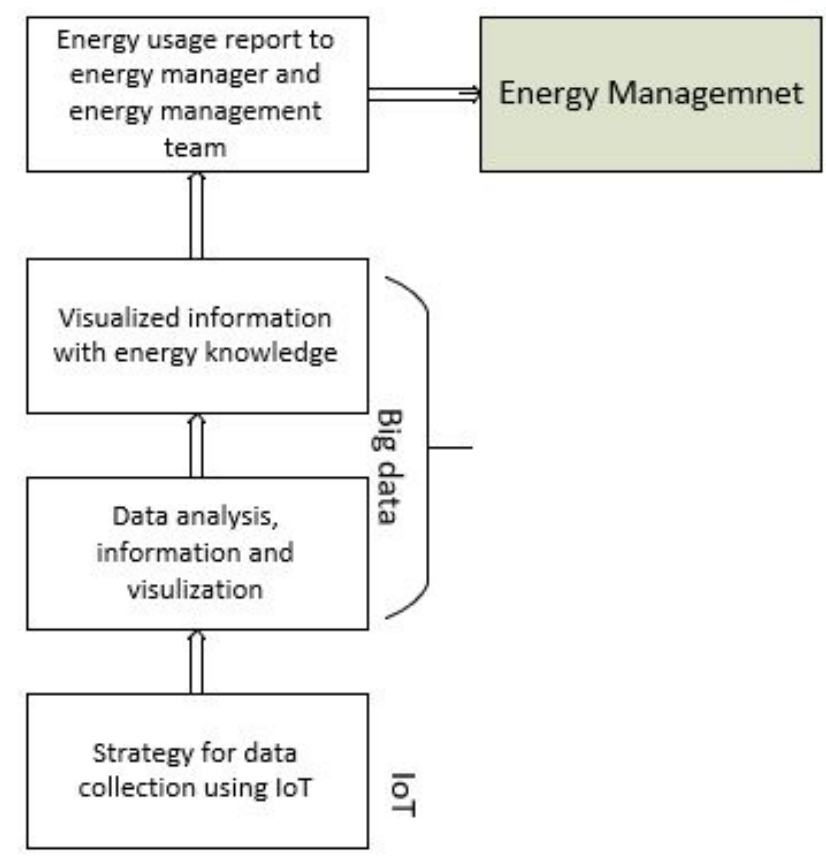

Fig. 2. Industrial Energy Management System

IoT and can be defined as small and complex systems that allow businesses, governments, and citizens to adopt and interconnect physical objects and virtual objects based on existing and evolving interoperable information and communication technologies [5]. IoT is a new technology paradigm that has emerged as a global network of machines and devices capable of interacting with each other and with the platform for collecting, analyzing, storing and visualizing data generated from the devices and machines using sensors, actuators, communications, and analytical tools [12]. IoT is now playing a main role in Industrial sector by collecting data from machines and other sources [13] and IoT platform is used to store, process and perform analytics on the collected data and provide useful information to be sent to the customer, as shown in Figure 3.

An IoT platform provides services to IoT devices and customers and enables IoT device an endpoint management, connectivity and network management, processing and analysis, data management, application development, access control, security, monitoring, event processing and interfacing [5]. Recent increase and development in mobile devices, embedded technologies, cloud computing and data analytics has resulted in a boom in IoT utilization, in terms of personal and organizational use, to conduct information exchange to facilitate recognition, monitoring, tracing, positioning and administration [5],[6]. The number of IoT platforms are increasing at a rapid speed, For example, in 2015 the number of IoT platforms were almost 260, which grew to 360 in 2016, exceeded to 450 in 2017 and reached to 620 in 2019 [14]. Requirements for IoT platforms, which provide important services and features for IoT applications, change as new IoT devices emerge [9]. This complexity in the context of rapid change poses

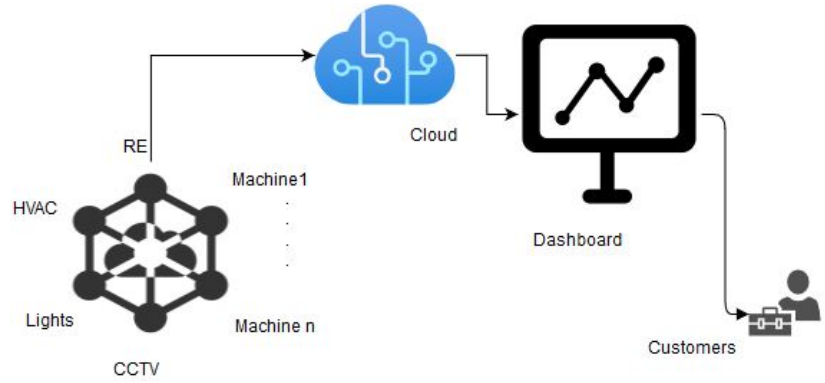

Fig. 3. IoT for IEnMS

challenges for businesses, governments and citizens that often have little experience of the infrastructure of IoT and limited knowledge of how to select an IoT platform that can meet their current and future needs.

IoT application needs a platform to run smoothly and provide the data so that the companies take present and future business decisions based on the data received from the IoT platform [11]. An IoT platform is the main part of an IoT solutions. Among the hundreds of IoT platform vendors in the market, it becomes very difficult for the companies to find and select a suitable IoT platform that is best suitable for their business application and which can fulfil their maximum business requirements. This problem can be solved by two steps. In the first step, the company can identify their complete present and future business requirements. In the second step the company should have the knowledge of some of the key factors of an IoT platform before selection. In this way the company can select a suitable IoT platform for their business application by comparing the requirements with their features.

\section{GENERAL FRAMEWORK FOR SELECTION OF IOT PLATFORM FOR IENMS}

We have aimed to create a more general approach that can be more widely used across all the cases. To show how our general framework can be applied to assessing and choosing an IoT platforms, in this study we have selected the top five IoT platforms (AWS, Azure, Google, IBM, and Oracle) based on market share. We have compared these IoT platforms according to the twenty-one key IoT platform factors that we have identified in [5]. We have compared these twenty-one key factors with the features provided by those selected five IoT platforms as shown in Table II.

More specifically, the entries of Table II have the following meaning related to the specific feature to be considered: 'yes' means the feature is available, 'high' indicates strong, 'bad' shows weak, 'good' indicates that the feature is very good, '-' shows that the feature is unknown and 'no' indicates that the feature is not available in the platform. In order to identify and fill the features of the selected five IoT platforms, different articles [5], [15]-[20] have been studied from many databases. Some websites [21]-[26] have been used, especially the websites of those selected IoT platforms. A few white papers [27] have also been studied. 

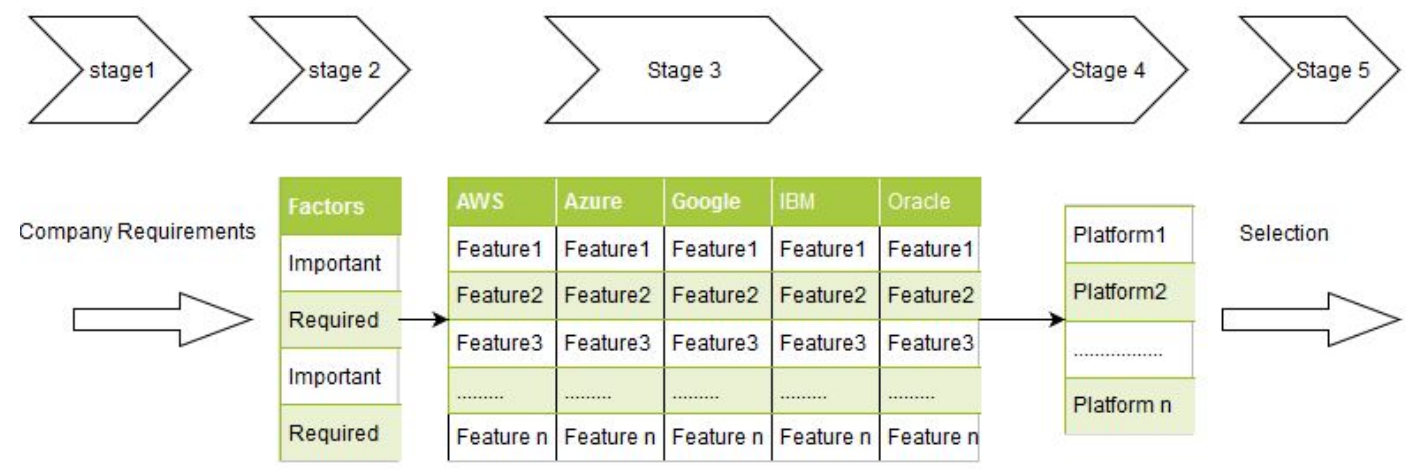

Fig. 4. IoT platform selection Framework for Industrial Energy Management System

TABLE II

FEATURES PROVIDED BY IOT PLATFORMS [13]

\begin{tabular}{lccccc}
\hline Factors & AWS & Azure & Google & IBM & Oracle \\
\hline Scalability & Yes & Yes & Yes & Yes & Yes \\
\hline Flexibility & Yes & - & Yes & - & Yes \\
\hline Stability & Yes & Yes & Yes & - & - \\
\hline Security & High & High & High & High & High \\
\hline Data analytics & Yes & Yes & Yes & Yes & Yes \\
\hline Disaster recovery & Yes & Yes & No & No & No \\
\hline Data ownership & - & Yes & - & - & - \\
\hline Protocol support & Yes & Yes & - & Yes & Yes \\
\hline System performance & Yes & - & Yes & Yes & - \\
\hline Interoperability & Yes & - & - & - & Yes \\
\hline App. environment & Yes & Yes & Yes & Yes & Yes \\
\hline Cloud ownership & Yes & Yes & Yes & & Yes \\
\hline Pricing model & Bad & Bad & Good & - & - \\
\hline Legacy architecture & Yes & - & - & - & Yes \\
\hline Attractive interface & Yes & Yes & - & No & - \\
\hline Time to market & Yes & Yes & & & Yes \\
\hline Bandwidth & - & - & Good & - & - \\
\hline Edge intelligence & Yes & Yes & Yes & - & Yes \\
\hline Hybrid cloud & Yes & Yes & - & - & - \\
\hline Platform migration & Yes & Yes & - & - & - \\
\hline Previous experience & Yes & Yes & - & - & - \\
\hline
\end{tabular}

The framework for selection of an IoT platform is illustrated in Fig.4. as a schematic of the selection procedure. The whole process consists of five stages. In the first stage, the company finalize their business requirements. In the second stage, the company requirements are applied to prioritising which factors are required $(\mathrm{R})$ and important (I) for this business context. In the third stage, the R and I factors are compared with the features provided by the five selected IoT platforms. The IoT platform/s that provide a maximum of the features as compared to the requirements are selected and shifted to the stage four. In stage four, there might be one or many IoT platforms that match the required and important factors. Stage five is the decision, which is explained next.

If there is one IoT platform that provides the most required and important features then the same IoT platform can be selected for the business application. But, if there are multiple IoT platforms providing these features then the company may choose an IoT platform based on the comparison of their match to " $\mathrm{S}$ " factors and select a suitable IoT platform for their business needs. There might also be chances that none of the platforms provide all of the required features; this might indicate that new platforms should be selected and evaluated accordingly.

For the IEnMS, the five stages of the framework are explained in a simple way. A company is interested to start energy management system to save reduce greenhouse gas emission, save energy and reduce energy bills. The company is interested to use IoT application for its EnMS. Initially the have to learn the components of IoT to understand what is IoT and how it works. Secondly they have to study to know what is an IoT platform and what are key factors of an IoT platform. They need an IoT platform for their business application but they do not know which platforms are providing what features and which one is best. when the company have the knowledge of IoT, IoT platform factors and the features those platforms are providing then in stage 1 , the company go through each of the 21 factors that have been identified as important in choosing a platform and use this to help them to formulate their business requirements. In stage 2 , these factors are prioritized as being either required (R) and important (I) for their business needs. They find that their required factors to consider (R) are for example, scalability, stability, system performance, attractive interface, edge intelligence, time to market, flexibility, and previous experience. Their important factors to consider (I) are for example pricing, security, data analytics, disaster recovery, and interoperability. In stage 3, the $\mathrm{R}$ and I factors are compared with identified features of IoT platforms. The platform that is fulfilling the "I" and "R" requirements of the company can be selected. In case there are multiple of IoT platforms that are offering completely the "I" and "R" requirements of the company, then the company can select one that is providing the "I" requirements in a better way. In some cases it can happen that non of the IoT platform are providing all the "I" and "R" requirements, in this case the company can search some other IoT platform for their IoT application.

\section{Conclusions}

The aim of this study is to build an objective methodology that can support industries to select the most suitable IoT platform for their IEnMS based on their specific needs. To do so, we first highlight the components of EnM and later we have explained the components and working of IEnMS. Second, we identified twenty-one key factors of IoT platforms from the literature. Finally, 
we have designed a theoretical framework for selection of IoT platform for IEnMS and tested it in five wellknown examples. This research then provides a general framework to select the most suitable IoT platform for industries to build their IEnMS by comparing its specific requirements with the features offered by the different platforms.

We believe industries can select an appropriate IoT platform for their IoT application, if they first analyze their business requirements, and start selection of the IoT platform with clear business requirements and have the knowledge of key factors of IoT platforms. This study highlighted important key factors of IoT platform. Those key factors may cover some of the current requirements of the IEnMS and can help ensure that current and future needs of the business are meet. It will facilitate the companies to select a suitable IoT platform for their business needs.

\section{ACKNOWLEDGEMENTS}

This paper is supported by Academy of Finland via: (a) ee-IoT project n.319009, (b) FIREMAN consortium CHIST-ERA/n.326270, and (c) EnergyNet Research Fellowship n.321265/n.328869.

\section{REFERENCES}

[1] E. Pinero, "Iso 50001: setting the standard for industrial energy management," Green Manufacturing News, pp. 21-24, 2009.

[2] A. Sa, P. Thollander, and E. Cagno, "Assessing the driving factors for energy management program adoption," Renewable and Sustainable Energy Reviews, vol. 74, pp. 538-547, 2017.

[3] M. Wei, S. H. Hong, and M. Alam, "An iot-based energymanagement platform for industrial facilities," Applied energy, vol 164, pp. 607-619, 2016.

[4] M. Ullah, S. R. U. Kakakhel, T. Westerlund, A. Wolff, D. Carrillo, J. Plosila, and P. H. Nardelli, "Iot protocol selection for smart grid applications: Merging qualitative and quantitative metrics," in 2020 $43 r$ International Convention on Information, Communication and Electronic Technology (MIPRO). IEEE, 2020, pp. 993-998.

[5] M. Ullah and K. Smolander, "Highlighting the Key Factors of an IoT Platform," 2019 42nd International Convention on Information and Communication Technology, Electronics and Microelectronics (MIPRO), pp. 901-906, 2019.

[6] B. Purwanggono, K. Ferastra, and A. Bachtiar, "Critical success factors evaluation of the iso 50001 energy management system implementation (case study: Pt. apac inti corpora, bawen, semarang indonesia)," in IOP Conference Series: Materials Science and Engineering, vol. 598, no. 1. IOP Publishing, 2019, p. 012115.

[7] M. Schulze, H. Nehler, M. Ottosson, and P. Thollander, "Energy management in industry-a systematic review of previous findings and an integrative conceptual framework," Journal of cleaner production, vol. 112, pp. 3692-3708, 2016

[12] M. Ullah, A. Hekmatmanesh, D. Savchenko, R. Moioli, P. Nardelli, $\mathrm{H}$. Handroos, and $\mathrm{H}$. Wu, "Providing facilities in health care via brain-computer interface and internet of things," in 2020 43rd International Convention on Information, Communication and Electronic Technology (MIPRO). IEEE, 2020, pp. 971-976.
[8] L. Sivill, J. Manninen, I. Hippinen, and P. Ahtila, "Success factors of energy management in energy-intensive industries: Development priority of energy performance measurement," International journal of energy research, vol. 37, no. 8, pp. 936-951, 2013.

[9] E. V. Tachmitzaki, E. A. Didaskalou, and D. A. Georgakellos, "Energy management practices' determinants in greek enterprises," Sustainability, vol. 12, no. 1, p. 133, 2020

[10] A. Sa, P. Thollander, and M. Rafiee, "Industrial energy management systems and energy-related decision-making," Energies, vol. 11, no. 10, p. 2784, 2018.

[11] V. A. da Silva Gonçalves and F. J. M.-H. dos Santos, "Energy management system iso 50001: 2011 and energy management for sustainable development," Energy Policy, vol. 133, p. 110868, 2019.

[13] M. Ullah, P. H. Nardelli, A. Wolff, and K. Smolander, "Twenty-one key factors to choose an iot platform: Theoretical framework and its applications," IEEE Internet of Things Journal, vol. 7, no. 10, pp. 10111-10119, 2020

[14] S. Liu, "• Number of IoT platforms worldwide 2015-2019 Statista." [Online]. Available: https://www.statista.com/statistics/ 1101483/global-number-iot-platform/

[15] P. P. Ray, "A survey of IoT cloud platforms," Future Computing and Informatics Journal, vol. 1, no. 1-2, pp. 35-46, 2017. [Online]. Available: http://dx.doi.org/10.1016/j.fcij.2017.02.001

[16] P. Agarwal and M. Alam, "Investigating IoT Middleware Platforms for Smart Application Development," pp. 1-14, 2018. [Online]. Available: http://arxiv.org/abs/1810.12292

[17] H. Hejazi, H. Rajab, T. Cinkler, and L. Lengyel, "Survey of platforms for massive IoT," 2018 IEEE International Conference on Future IoT Technologies, Future IoT 2018, vol. 2018-Janua, pp. $1-8,2018$.

[18] M. Ammar and B. Crispo, "slimIoT : Scalable Lightweight Attestation Protocol For the Internet of Things."

[19] S. Challita, F. Zalila, C. Gourdin, and P. Merle, "A precise mode for Google cloud platform," Proceedings - 2018 IEEE International Conference on Cloud Engineering, IC2E 2018, pp. 177-183, 2018.

[20] B. Nakhuva and T. Champaneria, "Study of Various Internet of Things Platforms," International Journal of Computer Science \& Engineering Survey, vol. 6, no. 6, pp. 61-74, 2015.

[21] Amazon Web Services, "IoT Applications \& Solutions - What is the Internet of Things (IoT)? - AWS," 2019. [Online]. Available: https://aws.amazon.com/iot/

[22] Google Cloud, "Google Cloud IoT - Fully managed IoT services - Google Cloud," 2019. [Online]. Available: https: //cloud.google.com/solutions/iot/

[23] IBM Watson IoT Platform, "IBM Watson IoT Platform - Overview - Finland," 2019. [Online]. Available: https://www.ibm.com/fi-en/ marketplace/internet-of-things-cloud

[24] Microsoft Azure IoT, "Microsoft Azure," 2019. [Online]. Available: https://azure.microsoft.com/en-in/pricing/details/iot-hub/

[25] Internet of Things, "Internet of Things (IoT) - Oracle," 2019 [Online]. Available: https://www.oracle.com/internet-of-things/

[26] IOTIFY, "Top 10 criteria to choose the best IoT cloud platform." [Online]. Available: https://iotify.io/ top-10-selection-criteria-for-your-iot-cloud-platform/

[27] G. Cloud, I. Security, D. Overview, G. Cloud, G. Cloud, A. Layer, and T. Security, "Google Cloud Security Whitepapers," no. March, pp. 1-97, 2018. 